\title{
Phenomenology of Sivers Effect with TMD Evolution
}

\author{
Mauro Anselmino ${ }^{1}$, Mariaelena Boglione ${ }^{1}$, Stefano Melis ${ }^{2}$ \\ 1 Dipartimento di Fisica Teorica, Università di Torino, \\ and INFN - Sezione di Torino, Via P. Giuria 1, I-10125 Torino, Italy \\ 2 European Centre for Theoretical Studies in Nuclear Physics and Related Areas (ECT*), \\ Villa Tambosi, Strada delle Tabarelle 286, I-38123 Villazzano, Trento, Italy
}

DOI: http://dx.doi.org/10.3204/DESY-PROC-2012-02/256

\begin{abstract}
Following the TMD evolution scheme recently proposed for the unpolarized and the Sivers distribution function, we propose a simple strategy to take into account this TMD $Q^{2}$ dependence in our phenomenological extraction of the Sivers function from SIDIS data. New results are presented and possible future applications are discussed.
\end{abstract}

The exploration of the 3-dimensional structure of the nucleon, both in momentum and in configuration space, is one of the major issues in high energy hadron physics. Information on this 3-dimensional structure is embedded in the Transverse Momentum Dependent distribution and fragmentation functions (TMDs). The Sivers function, which describes the number density of unpolarized quarks inside a transversely polarized proton, is particularly interesting, as it might provide information on the partonic orbital angular momentum.

So far, all phenomenological fits have either neglected the QCD scale dependence of TMDs (which was unknown) or limited it to the collinear part of the unpolarized PDFs, according to the DGLAP evolution. Here, we present the first attempt to take into account the TMD evolution as proposed by Aybat, Collins, Qiu and Rogers [1, 2, 3] in the analysis of the Sivers asymmetry data and show how these new results compare with the previous extractions. Eventually, such a scheme will provide a complete TMD factorization framework for a consistent treatment of all SIDIS data.

In Ref. [4] we showed how the QCD evolution equation of the TMDs in the coordinate space proposed in Refs. 2] and 3] can be expressed in a simplified way, taking the renormalization scale $\mu^{2}$ and the regulating parameters $\zeta_{F}$ and $\zeta_{D}$ all equal to $Q^{2}$, as

$$
\widetilde{F}\left(x, \boldsymbol{b}_{T} ; Q\right)=\widetilde{F}\left(x, \boldsymbol{b}_{T} ; Q_{0}\right) \widetilde{R}\left(Q, Q_{0}, b_{T}\right) \exp \left\{-g_{K}\left(b_{T}\right) \ln \frac{Q}{Q_{0}}\right\},
$$

where $\widetilde{F}$ can be either the unpolarized parton distribution, $\widetilde{F}\left(x, \boldsymbol{b}_{T} ; Q\right)=\widetilde{f}_{q / p}\left(x, \boldsymbol{b}_{T} ; Q\right)$, the unpolarized fragmentation function $\widetilde{F}\left(x, \boldsymbol{b}_{T} ; Q\right)=\widetilde{D}_{h / q}\left(z, \boldsymbol{b}_{T} ; Q\right)$, or the first derivative, with respect to the parton impact parameter $b_{T}$, of the Sivers function, $\widetilde{F}\left(x, \boldsymbol{b}_{T} ; Q\right)=\tilde{\tilde{f}_{1 T}^{\prime} \perp f}\left(x, \boldsymbol{b}_{T} ; Q\right)$; $g_{K}\left(b_{T}\right)$ is an unknown, but universal and scale independent, input function, while $\widetilde{R}\left(Q, Q_{0}, b_{T}\right)$ is the evolution kernel

$$
\widetilde{R}\left(Q, Q_{0}, b_{T}\right) \equiv \exp \left\{\ln \frac{Q}{Q_{0}} \int_{Q_{0}}^{\mu_{b}} \frac{\mathrm{d} \mu^{\prime}}{\mu^{\prime}} \gamma_{K}\left(\mu^{\prime}\right)+\int_{Q_{0}}^{Q} \frac{\mathrm{d} \mu}{\mu} \gamma_{F}\left(\mu, \frac{Q^{2}}{\mu^{2}}\right)\right\} .
$$


The anomalous dimensions $\gamma_{F}$ and $\gamma_{K}$ appearing in Eq. 22], are given, at order $\mathcal{O}\left(\alpha_{s}\right)$, by [2]

$$
\gamma_{F}\left(\mu ; \frac{Q^{2}}{\mu^{2}}\right)=\alpha_{s}(\mu) \frac{C_{F}}{\pi}\left(\frac{3}{2}-\ln \frac{Q^{2}}{\mu^{2}}\right) \quad \gamma_{K}(\mu)=\alpha_{s}(\mu) \frac{2 C_{F}}{\pi} .
$$

The $Q^{2}$ evolution is therefore driven by the functions $g_{K}\left(b_{T}\right)$ and $\widetilde{R}\left(Q, Q_{0}, b_{T}\right)$. While the latter, Eq. (2), can be easily evaluated, numerically or even analytically, the former, is essentially unknown and will need to be taken from independent experimental inputs.

The appropriate Fourier transforms allow us to obtain the distribution and fragmentation functions in the momentum space:

$$
\begin{aligned}
& \widehat{f}_{q / p}\left(x, k_{\perp} ; Q\right)=\frac{1}{2 \pi} \int_{0}^{\infty} \mathrm{d} b_{T} b_{T} J_{0}\left(k_{\perp} b_{T}\right) \widetilde{f}_{q / p}\left(x, b_{T} ; Q\right), \\
& \widehat{D}_{h / q}\left(z, p_{\perp} ; Q\right)=\frac{1}{2 \pi} \int_{0}^{\infty} \mathrm{d} b_{T} b_{T} J_{0}\left(\mathrm{k}_{T} b_{T}\right) \widetilde{D}_{h / q}\left(z, b_{T} ; Q\right), \\
& \widehat{f}_{1 T}^{\perp f}\left(x, k_{\perp} ; Q\right)=\frac{-1}{2 \pi k_{\perp}} \int_{0}^{\infty} \mathrm{d} b_{T} b_{T} J_{1}\left(k_{\perp} b_{T}\right) \widetilde{f}_{1 T}^{\prime \perp q}\left(x, b_{T} ; Q\right),
\end{aligned}
$$

where $J_{0}$ and $J_{1}$ are Bessel functions, while $\widehat{f}_{q / p}$ is the unpolarized TMD distribution function for a parton of flavor $q$ inside a proton, $\widehat{D}_{h / q}$ is the unpolarized TMD fragmentation function for hadron $h$ inside a parton $q$ and $\widehat{f}_{1 T}^{\perp q}$ is the Sivers distribution describing unpolarized partons inside a transversely polarized proton.

The unknown input functions $g_{K}\left(b_{T}\right)$ and $\widetilde{F}\left(x, b_{T} ; Q_{0}\right)$ inside Eq. (1) have to be appropriately parameterized. As already anticipated, $g_{K}\left(b_{T}\right)$ is a non-perturbative, but universal function, which in the literature is usually parameterized in a quadratic form: $g_{K}\left(b_{T}\right)=\frac{1}{2} g_{2} b_{T}^{2}$. As in Ref. [3] we will adopt the results provided by a recent fit of Drell-Yan data [5], and assume $g_{2}=0.68 \mathrm{GeV}^{2}$. The input functions $\widetilde{F}\left(x, b_{T} ; Q_{0}\right)$ are parameterized by requiring that their Fourier-transforms, which give the corresponding TMD functions in the transverse momentum space, coincide with the previously adopted $k_{\perp}$-Gaussian forms, with the $x$ dependence factorized out. As shown in Ref. 4, one finds

$$
\begin{aligned}
& \widetilde{f}_{q / p}\left(x, b_{T} ; Q\right)=f_{q / p}\left(x, Q_{0}\right) \widetilde{R}\left(Q, Q_{0}, b_{T}\right) \exp \left\{-b_{T}^{2}\left(\alpha^{2}+\frac{g_{2}}{2} \ln \frac{Q}{Q_{0}}\right)\right\} \\
& \widetilde{D}_{h / q}\left(z, b_{T} ; Q\right)=\frac{1}{z^{2}} D_{h / q}\left(z, Q_{0}\right) \widetilde{R}\left(Q, Q_{0}, b_{T}\right) \exp \left\{-b_{T}^{2}\left(\beta^{2}+\frac{g_{2}}{2} \ln \frac{Q}{Q_{0}}\right)\right\} \\
& \widetilde{f}_{1 T}^{\prime \perp}\left(x, b_{T} ; Q\right)=-2 \gamma^{2} f_{1 T}^{\perp}\left(x ; Q_{0}\right) \widetilde{R}\left(Q, Q_{0}, b_{T}\right) b_{T} \exp \left\{-b_{T}^{2}\left(\gamma^{2}+\frac{g_{2}}{2} \ln \frac{Q}{Q_{0}}\right)\right\}
\end{aligned}
$$

with $\alpha^{2}=\left\langle k_{\perp}^{2}\right\rangle / 4, \beta^{2}=\left\langle p_{\perp}^{2}\right\rangle /\left(4 z^{2}\right), 4 \gamma^{2} \equiv\left\langle k_{\perp}^{2}\right\rangle_{S}=\frac{M_{1}^{2}\left\langle k_{\perp}^{2}\right\rangle}{M_{1}^{2}+\left\langle k_{\perp}^{2}\right\rangle}$, and $\widetilde{R}\left(Q, Q_{0}, b_{T}\right)$ in Eq. 22.

Eqs. (7)-(9) show that the $Q^{2}$ evolution is controlled by the logarithmic $Q$ dependence of the $b_{T}$ Gaussian width, together with the factor $\widetilde{R}\left(Q, Q_{0}, b_{T}\right)$ : for increasing values of $Q^{2}$, they are responsible for the typical broadening effect already observed in Refs. [2] and [3].

As $R\left(Q, Q_{0}, b_{T}\right)$ shows a weak dependence on (large) $b_{T}$ (i.e. small $k_{\perp}$ ), we can assume $R\left(Q, Q_{0}, b_{T}\right)$ to be constant in $b_{T}$ and compute the Fourier transforms of the evolution equa- 
Table 1: $\chi^{2}$ contributions corresponding to our three fits, for some experimental data sets of HERMES and COMPASS experiments.

\begin{tabular}{cccc}
\hline & TMD Evolution (exact) & TMD Evolution (analyt.) & DGLAP Evolution \\
\cline { 2 - 4 } & $\chi_{\text {tot }}^{2}=255.8$ & $\chi_{\text {tot }}^{2}=275.7$ & $\chi_{\text {tot }}^{2}=315.6$ \\
$\chi_{\text {d.o.f }}^{2}=1.02$ & $\chi_{d . o . f}^{2}=1.10$ & $\chi_{\text {d.o.f }}^{2}=1.26$ \\
\hline \multirow{3}{*}{ HERMES $\pi^{+}$} & $\chi_{x}^{2}=10.7$ & $\chi_{x}^{2}=12.9$ & $\chi_{x}^{2}=27.5$ \\
& $\chi_{z}^{2}=4.3$ & $\chi_{z}^{2}=4.3$ & $\chi_{z}^{2}=8.6$ \\
& $\chi_{P_{T}}^{2}=9.1$ & $\chi_{P_{T}}^{2}=10.5$ & $\chi_{P_{T}}^{2}=22.5$ \\
\hline \multirow{2}{*}{ COMPASS $h^{+}$} & $\chi_{x}^{2}=6.7$ & $\chi_{x}^{2}=11.2$ & $\chi_{x}^{2}=29.2$ \\
& $\chi_{z}^{2}=17.8$ & $\chi_{z}^{2}=18.5$ & $\chi_{z}^{2}=16.6$ \\
& $\chi_{P_{T}}^{2}=12.4$ & $\chi_{P_{T}}^{2}=24.2$ & $\chi_{P_{T}}^{2}=11.8$ \\
\hline
\end{tabular}

tions (7), (8) and (9) analytically, to find

$$
\begin{aligned}
\widehat{f}_{q / p}\left(x, k_{\perp} ; Q\right) & =f_{q / p}\left(x, Q_{0}\right) R\left(Q, Q_{0}\right) \frac{e^{-k_{\perp}^{2} / w^{2}}}{\pi w^{2}} \\
\widehat{D}_{h / q}\left(z, p_{\perp} ; Q\right) & =D_{h / q}\left(z, Q_{0}\right) R\left(Q, Q_{0}\right) \frac{e^{-p_{\perp}^{2} / w_{F}^{2}}}{\pi w_{F}^{2}} \\
\Delta^{N} \widehat{f}_{q / p^{\uparrow}}\left(x, k_{\perp} ; Q\right) & =\frac{k_{\perp}}{M_{1}} \sqrt{2 e} \frac{\left\langle k_{\perp}^{2}\right\rangle_{S}^{2}}{\left\langle k_{\perp}^{2}\right\rangle} \Delta^{N} f_{q / p^{\uparrow}}\left(x, Q_{0}\right) R\left(Q, Q_{0}\right) \frac{e^{-k_{\perp}^{2} / w_{S}^{2}}}{\pi w_{S}^{4}},
\end{aligned}
$$

where $f_{q / p}\left(x, Q_{0}\right)$ and $D_{h / q}\left(z, Q_{0}\right)$ are the usual integrated PDF evaluated at the initial scale $Q_{0}$, and $\Delta^{N} f_{q / p^{\uparrow}}\left(x, Q_{0}\right)$ gives the $x$ dependence of the Sivers function [4] [- $\left(2 k_{\perp} / M_{p}\right) \widehat{f}_{1 T}^{\perp}=$ $\left.\Delta^{N} \widehat{f}_{q / p^{\uparrow}}\right]$. Most importantly, $w^{2}, w_{F}^{2}$ and $w_{S}^{2}$ are the "evolving" Gaussian widths, defined as:

$$
w^{2}=\left\langle k_{\perp}^{2}\right\rangle+2 g_{2} \ln \frac{Q}{Q_{0}}, \quad w_{F}^{2}=\left\langle p_{\perp}^{2}\right\rangle+2 z^{2} g_{2} \ln \frac{Q}{Q_{0}}, \quad w_{S}^{2}=\left\langle k_{\perp}^{2}\right\rangle_{S}+2 g_{2} \ln \frac{Q}{Q_{0}} .
$$

Notice that the $Q^{2}$ evolution of the TMD PDFs is now determined by the overall factor $R\left(Q, Q_{0}\right)$ and, most crucially, by the $Q^{2}$ dependent Gaussian width $w\left(Q, Q_{0}\right)$.

It is interesting to point that the evolution factor $R\left(Q, Q_{0}\right)$, controlling the TMD evolution, is the same for all functions (TMD PDFs, TMD FFs and Sivers ) and is flavor independent: consequently it will appear, squared, in both numerator and denominator of the Sivers azimuthal asymmetry and, approximately, cancel out. Therefore, we can safely conclude that most of the TMD evolution of azimuthal asymmetries is controlled by the logarithmic $Q$ dependence of the $k_{\perp}$ Gaussian widths $w^{2}\left(Q, Q_{0}\right)$, Eq. [13).

The aim of our paper is to analyze the available polarized SIDIS data from the HERMES and COMPASS collaborations in order to understand whether or not they show signs of the TMD evolution proposed in Ref. [3]. In particular we perform three different data fits of the SIDIS Sivers single spin asymmetry $A_{U T}^{\sin \left(\phi_{h}-\phi_{S}\right)}$ measured by HERMES and COMPASS: a fit (TMD-fit) in which we adopt the TMD evolution equations of Eqs. (7)-(9); second fit (TMDanalytical-fit) in which we apply the same TMD evolution, but using the analytical approximation of Eqs. (10), (11) and (12); a fit (DGLAP-fit) in which we follow our previous work, as done so far in Ref. [6, 7], using the DGLAP evolution equation only in the collinear part of the TMDs. 
Table I shows the main results of our fitting procedure. The best total $\chi_{\text {tot }}^{2}$, which amounts to 256 , is obtained by using the TMD evolution, followed by a slightly higher $\chi_{\text {tot }}^{2}$ of the analytical approximation, and a definitely larger $\chi_{\text {tot }}^{2} \simeq 316$ corresponding to the DGLAP fit. The difference of about 60 $\chi^{2}$-points between the TMD and the DGLAP fits is heavily concentrated in the asymmetry for $\pi^{+}$production at HERMES and for $h^{+}$production at COMPASS, especially when this asymmetry is observed as a function of the $x$-variable. It is important to stress that, as $x$ is directly proportional to $Q^{2}$ through the kinematical relation $Q^{2}=x y s$, the $x$ behavior of the asymmetries is intimately connected to their $Q^{2}$ evolution. While the HERMES experimental bins cover a very modest range of $Q^{2}$ values, from $1.3 \mathrm{GeV}^{2}$ to $6.2 \mathrm{GeV}^{2}$, COMPASS data raise to a maximum $Q^{2}$ of
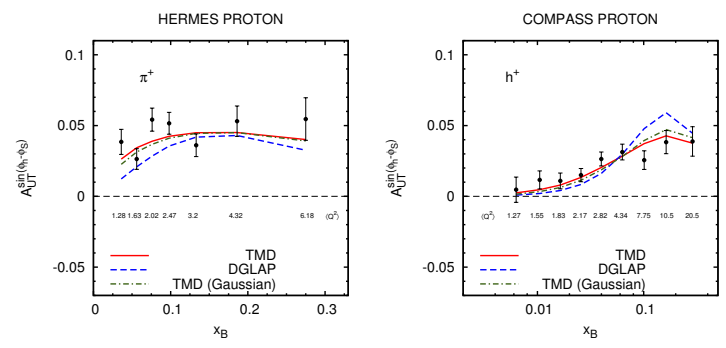

Figure 1: The results obtained from our fit of the SIDIS $A_{U T}^{\sin \left(\phi_{h}-\phi_{S}\right)}$ Sivers asymmetries applying TMD evolution (red, solid lines) are compared with the analogous results found by using DGLAP evolution equations (blue, dashed lines). The green, dash-dotted lines correspond to the results obtained by using the approximated analytical TMD evolution. The experimental data are from HERMES [8] (left panel) and COMPASS [9] (right panel) Collaborations. $20.5 \mathrm{GeV}^{2}$, enabling to test more severely the TMD $Q^{2}$ evolution in SIDIS. These aspects are illustrated in Fig. 1, where the SIDIS Sivers asymmetries $A_{U T}^{\sin \left(\phi_{h}-\phi_{S}\right)}$ obtained in the three fits are shown in the same plot. It is evident that the DGLAP evolution seems to be unable to describe the correct $x$ trend, i.e. the right $Q^{2}$ behavior, while the TMD evolution (red solid line) follows much better the large $Q^{2}$ data points, corresponding to the last $x$-bins measured by COMPASS.

In conclusions, we have analyzed the Sivers effect by up-grading old fits with the addition of the most recent HERMES and COMPASS SIDIS data, and by applying, for the first time, TMD evolution equations. We have compared the results obtained using TMD evolution equations with those found by considering only the DGLAP evolution of the collinear part of the TMDs. Our results give evidence that SIDIS data support the TMD evolution scheme, although further experimental data, covering a wider range of $Q^{2}$ values, are necessary to confirm this.

\section{References}

[1] J. C. Collins, Foundations of Perturbative QCD, Cambridge Monographs on Particle Physics, Nuclear Physics and Cosmology, No. 32, Cambridge University Press, Cambridge, 2011.

[2] S. M. Aybat and T. C. Rogers, Phys. Rev. D 83, 114042 (2011) [arXiv:1101.5057 [hep-ph]].

[3] S. M. Aybat, J. C. Collins, J. -W. Qiu and T. C. Rogers, arXiv:1110.6428 [hep-ph].

[4] M. Anselmino, M. Boglione and S. Melis, arXiv:1204.1239 [hep-ph].

[5] F. Landry, R. Brock, P. M. Nadolsky and C. P. Yuan, Phys. Rev. D 67, 073016 (2003) [hep-ph/0212159].

[6] M. Anselmino, M. Boglione, U. D'Alesio, A. Kotzinian, S. Melis, F. Murgia, A. Prokudin and C. Türk, Eur. Phys. J. A 39, 89 (2009) [arXiv:0805.2677 [hep-ph]].

[7] M. Anselmino, M. Boglione, U. D'Alesio, S. Melis, F. Murgia and A. Prokudin, arXiv:1107.4446 [hep-ph].

[8] A. Airapetian et al. [HERMES Collaboration], Phys. Rev. Lett. 103, 152002 (2009) [arXiv:0906.3918 [hep-ex]].

[9] F. Bradamante [COMPASS Collaboration], arXiv:1111.0869 [hep-ex]. 